\title{
Erratum: Simulation on a Photocathode-based Microtron Using a 3D PIC Code
}

\author{
[J. Korean Phys. Soc. 66, 363 (2017)] \\ Sunjeong PARK, Young Uk JeOng, ${ }^{*}$ Seong Hee PARK, Kyu-Ha JANG and Nikolay A. Vinokurov \\ Center for Quantum-beam-based Radiation Research, \\ Korea Atomic Energy Research Institute, Daejeon 305-353, Korea \\ Eun-San KIM \\ Department of Physics, Kyunpook National University, Daegu 702-701, Korea
}

DOI: $10.3938 / \mathrm{jkps} .71 .591$

One affiliation of Sunjeong Park was omitted. It should be added as "Department of Physics, Kyungpook National University, Daegu 702-701, Korea". Affiliation of Eun-San Kim had a typo. It should be corrected as "Department of Physics, Kyungpook National University, Daegu 702-701, Korea".

*E-mail: yujung@kaeri.re.kr; Fax: +82-42-868-2969 